\title{
Additional induction chemotherapy to concurrent chemotherapy and intensity-modulated radiotherapy with or without nimotuzumab in first-line treatment for locoregionally advanced nasopharyngeal carcinoma: a propensity score matched analysis
}

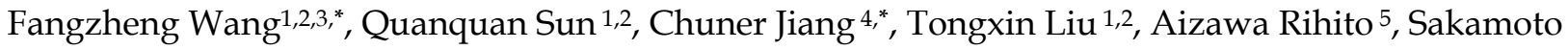
Masoto $^{3}$, Yuezhen Wang 1,2, Zhenfu Fu 1,2, Ming Chen 1,2凶

1. Department of Radiation Oncology, Zhejiang Cancer Hospital, Zhejiang Hangzhou, 310022, People's Republic of China.

2. Zhejiang Key Laboratory of Radiation Oncology, Zhejiang Hangzhou, 310022, People's Republic of China.

Department of Radiology, Japanese Red Cross Fukui Hospital, Fukui, 918-8501 Japan.

4. Department of Breast Tumor Surgery, Zhejiang Cancer Hospital, Zhejiang Hangzhou, 310022, People's Republic of China.

5. Department of Radiation Oncology and Image-Applied Therapy, Graduate School of Medicine, Kyoto University. Kyoto, 606-8507 Japan.

* These authors contributed equally to this work.

$\square$ Corresponding author: Ming Chen, Email: chenmingyy62@163.com

(c) Ivyspring International Publisher. This is an open access article distributed under the terms of the Creative Commons Attribution (CC BY-NC) license (https://creativecommons.org/licenses/by-nc/4.0/). See http://ivyspring.com/terms for full terms and conditions.

Received: 2017.04.07; Accepted: 2017.08.31; Published: 2018.01.01

\begin{abstract}
Background: The aim of this study is to assess the survival benefits of additional induction chemotherapy before concurrent chemotherapy, intensity-modulated radiotherapy and nimotuzumab in patients with locoregionally advanced nasopharyngeal carcinoma.

Methods: Clinical data from 1104 nonmetastatic nasopharyngeal carcinoma patients diagnosed between May 2008 and April 2014 were retrospectively reviewed. All patients received addition of induction chemotherapy to concurrent chemoradiotherapy with or without nimotuzumab. A propensity score matched method was used to identify paired patients according to various covariates.

Results: In total, 120 pairs were selected by propensity score matched method. At a median follow-up time of 56 months (10-99 months), the 5-year locoregional relapse-free survival, distant metastases-free survival, progression-free survival and overall survival rates in patients treated with nimotuzumab vs. without nimotuzumab were $91.6 \%$ vs. $91.1 \%(P=0.957), 95.8 \%$ vs. $83.9 \%(P=0.007), 87.4 \%$ vs. $81.3 \%(P=$ $0.225), 94.5 \%$ vs. $85.6 \%(P=0.058)$, respectively. Multivariate analysis revealed that nimotuzumab was an independent prognosticator of OS and DMFS.

Conclusions: Nimotuzumab is an effective treatment option for locoregionally advanced nasopharyngeal carcinoma, and the addition of induction chemotherapy to concurrent chemoradiotherapy and nimotuzumab could obtain the best survival benefits.
\end{abstract}

Key words: nasopharyngeal carcinoma, nimotuzumab, induction chemotherapy, concurrent chemotherapy, intensity-modulated radiotherapy, prognosis.

\section{Introduction}

The incidence of nasopharyngeal carcinoma (NPC) is 15 to 50 cases per 100,000 annually in Southern China, Singapore and Malaysia and it vary with age, ethnicity, and geographical origin [1].
Radiotherapy (RT) is the standard treatment for NPC because of the anatomical location and the high radiosensitivity. Patients with locoregionally advanced NPC at diagnosis account for $60 \%$ to $70 \%$ of 
all NPC patients [2]. Intensity modulated radiation therapy (IMRT) has been used to improve locoregional control but provides little benefit for survival outcome and prevention of distant failure [3, 4]. According to a meta-analysis of randomized studies, combination RT and chemotherapy reduces the risk of mortality by $18 \%$ and increases 5 -year survival by $4 \%$ to $6 \%$ [5]. Concurrent chemoradiotherapy (CCRT) with or without adjuvant chemotherapy, provides a benefit in overall survival and has become the standard treatment for locoregionally advanced NPC, although with acute toxicities [6-8]. A previous meta-analysis showed that compared with CCRT alone, addition of induction chemotherapy (IC) to CCRT reduces distant failure in locoregionally advanced NPC patients $[9,10]$, and another current meta-analysis confirmed that IC followed by CCRT significantly improved progression-free survival (PFS) and overall survival (OS) [11]. However, the efficacy of the additional IC to CCRT in patients with locoregionally advanced NPC remains controversial [12-14]. Considering these results, addition of IC to CCRT has been as a promising option for regionally advanced NPC patients in the era of IMRT. At present, the incidence of distant failure in locoregionally advanced NPC patients after combined treatment is more than $20 \%$ [15]. Therefore, new and effective regimens with tolerable toxicity for locoregionally advanced NPC are needed.

Overexpression of epidermal growth factor receptor (EGFR) is observed in many different cancers, including gliomas, sarcomas, and head and neck cancers [16]. Moreover, high EGFR expression is associated with poor prognosis [17, 18]. Several inhibitors of EGFR, such as cetuximab, panitumumab, erlotinib, and gefitinib, have shown favorable results in clinical trials $[19,20]$. Cetuximab, the most commonly used anti-EGFR antibody, combined with radiotherapy (RT), has been shown to improve survival in patients with locoregionally advanced head and neck squamous cell carcinoma [21]. In NPC, cetuximab with concurrent chemoradiotherapy is tolerable and has shown promising advantages for NPC prognosis [22]. However, the relatively high rates of mucositis and acne-like skin rash limit its clinical application [22, 23].

Nimotuzumab is a blocking anti-EGFR monoclonal antibody without intrinsic stimulating activity [24]. In the preclinical studies, nimotuzumab demonstrated antiproliferative, proapoptotic, and antiangiogenic activities [25], and nimotuzumab displayed a longer half-life and elevated area under the curve than cetuximab at the same dose level [26]. Nimotuzumab improves quality of life because it rarely causes severe dermatological toxicity, which is the most common adverse event resulting from cetuximab and panitumumab use [27].

Nimotuzumab has marketing approval for the treatment of locoregionally advanced NPC [28, 29]. However, the value of adding nimotuzumab to IC followed by CCRT in locoregionally advanced NPC patients remains unclear. Therefore, we performed this retrospective study to investigate long-term survival outcomes of nimotuzumab plus IC followed by CCRT in locoregionally advanced NPC patients. To avoid the interference of covariates, we used the propensity score matching (PSM) methods to select paired patients [30].

\section{Materials and methods}

\section{Patients}

The patients enrolled into this study were hospitalized in the Department of Radiation Oncology, Zhejiang Cancer Hospital between May 2008 to April 2014. The eligible patients met the following criteria: (i) histologically proven locoregionally advanced NPC, (ii) Eastern Cooperative Oncology Group performance status $\leq 1$, (iii) completion of radical IMRT, (iv) received IC before CCRT with or without nimotuzumab, and (v) no previous anti-cancer treatment. Ultimately, 240 subjects of 1104 patients were matched for the current study. This retrospective study was approved by the Medical Ethics Committee of Zhejiang Cancer Hospital. All the patients had signed the informed consent.

\section{Baseline examinations}

Patients had pretreatment evaluations that included complete histories, physical examinations, hematology and biochemistry profiles, chest radiographs, sonography of the abdomen, bone scans, magnetic response images of the nasopharynx, and nasopharyngoscopies. All patients were staged according to the 2010 American Joint Committee on Cancer staging system. Tumor histology was classified per the World Health Organization classification.

\section{Intensity-modulated radiotherapy}

All patients underwent radical IMRT with simultaneous integrated boost technique using $6 \mathrm{MV}$ photons with 2-3 weeks after IC. The delineation of target volumes of NPC during the treatment of IMRT was described previously [31]. Briefly, gross tumor volumes (GTV) of primary tumor and the metastatic lymph nodes were defined as GTVnx and GTVnd, which were delineated according to pre- and post-IC MR images, respectively. The clinical target volume of 
nasopharynx (CTVnx) was defined as GTVnx plus a 7 $\mathrm{mm}$ margin that encompassed the nasopharyngeal mucosa plus $5 \mathrm{~mm}$ submucosal volume. The high-risk clinical target volume (CTV1) included the entire nasopharyngeal cavity, the anterior one- to two-thirds of the clivus, the skull base, the pterygoid plates, the parapharyngeal space, the inferior sphenoid sinus, the posterior one-quarter to one-third of the nasal cavity, and the maxillary sinus and any lymph nodes in drainage pathways containing metastatic lymph nodes. The low-risk clinical target volume (CTV2) included levels IV and $\mathrm{Vb}$ without metastatic cervical lymph nodes.

The PTV was constructed automatically based on each volume with an additional 3-mm margin in three dimensions to account for set-up variability. All of the PTVs, including PGTVnx, PTVnx, PTV1, and PTV2, were not delineated outside of the skin surface. Critical normal structures including the brainstem, spinal cord, parotid glands, optic nerves, chiasm, lens, eyeballs, temporal lobes, temporomandibular joints, mandible, and hypophysis were contoured and set as OARs during optimization.

The prescribed radiation dose was 69 or $72 \mathrm{~Gy}$ to PGTVnx, 66-70 Gy to PGTVnd, 62-66 Gy to PTVnx, 60-63 Gy to PTV1, and 51-54 Gy to PTV2, delivered in 30 or 33 fractions. Radiation was delivered once daily, five fractions per week, over $6-6.5$ weeks for IMRT planning. The dose to OAR was limited on the basis of the RTOG 0225 protocol.

\section{Chemotherapy}

All patients were given two to four cycles of platinum-based induction chemotherapy. The available IC regimens included TPF (docetaxel 60 $\mathrm{mg} / \mathrm{m}^{2} /$ day on day 1 , cisplatin $25 \mathrm{mg} / \mathrm{m}^{2} /$ day on days 1 to 3 , and 5 -fluorouracil $500 \mathrm{mg} / \mathrm{m}^{2} /$ day on days 1 to 3 ), TP (docetaxel $60 \mathrm{mg} / \mathrm{m}^{2} /$ day on day 1 , cisplatin $25 \mathrm{mg} / \mathrm{m}^{2} /$ day on days 1 to 3 ), GP (gemcitabine $1,000 \mathrm{mg} / \mathrm{m}^{2} /$ day on days 1 and 8 , cisplatin $25 \mathrm{mg} / \mathrm{m}^{2} /$ day on days 1 to 3 ), and $\mathrm{FP}$ (cisplatin $25 \mathrm{mg} / \mathrm{m}^{2} /$ day on days 1 to 3 , and 5 -fluorouracil $500 \mathrm{mg} / \mathrm{m}^{2} /$ day on days 1 to 3 ).

Furthermore, the patients in this study underwent concurrent chemotherapy with cisplatin $\left(80 \mathrm{mg} / \mathrm{m}^{2}\right)$ divided to 3 days and received adjuvant chemotherapy with FP (cisplatin $25 \mathrm{mg} / \mathrm{m}^{2}$ /day on days $1-3$, and 5 -fluorouracil $500 \mathrm{mg} / \mathrm{m}^{2} /$ day on days 1-3) or GP regimens within 3-4 weeks after RT.

\section{Target treatment}

Nimotuzumab was administered concomitantly with CCRT at a dose of $200 \mathrm{mg}$ weekly, which was diluted in $250 \mathrm{~mL}$ of saline to obtain a 200-mg solution and it was intravenously infused over 1 hour. 120 patients received 6 to 17 weeks of nimotuzumab during the treatment of CCRT.

\section{Efficacy evaluation and follow-up}

The assessment of tumor response was performed thrice: after the completion of IC, at the end of IMRT and 3 months after irradiation. It was based on MRI and nasopharynx fiberscope according the Response Evaluation Criteria for Solid Tumors. Systemic chemotherapy adverse events were graded per the National Cancer Institute Common Toxicity Criteria (NCI CTCAE, Version 3.0), and RT-induced toxicities were scored using the Acute and Late Radiation Morbidity Scoring Criteria from the Radiation Therapy Oncology Group.

All the subjects underwent weekly examinations for treatment response and toxicities during RT. Patients were followed every 3 months in the first 2 years, every 6 months from the third to the fifth year, and then annually. Each follow-up included careful examination of the nasopharynx and neck nodes by an experienced doctor. MRI scans of the nasopharynx, nasopharynx fiberscopy, chest computed tomography radiography, and ultrasound of abdomen were performed 3 months after the completion of RT and every 6 to 12 months thereafter. Additional examinations were performed when indicated to evaluate local relapse or distant metastasis.

\section{Statistical analysis}

Survival curves were generated by use of the Kaplan-Meier method. The curves were compared using of log-rank tests. Multivariate analysis was performed by use of Cox regression models to identify significant prognostic factors. Hazard ratios (HRs) and 95\% confidence intervals (CIs) were calculated for each prognostic factor. IBM SPSS Statistics Version 19.0 was used for all data analysis. A $p<0.05$ was considered statistically significant. Survival time was calculated from the date of diagnosis to the most recent follow-up or to either the date of relapse (event-free, local recurrence-free, or distant metastasis-free) or death (overall survival). After recurrence or metastasis, patients were given salvage therapy as determined by their physicians.

\section{Results}

\section{Patient characteristics}

In total, the clinical data of 1104 newly diagnosed locoregionally advanced NPC patients, who were initially treated with IC followed by CCRT, were collected and retrospectively reviewed. From the original data, 120 pairs were selected by PSM. Basic characteristics of all patients are summarized in Table 1. For the selected subjects, the median age was 47 
years (range, 18-77 years), and the ratio of male to female was 2.08: 1 (162:78). There were no statistically significant differences in age, gender, stage and treatment factors between the nimotuzumab group and non-nimotuzumab group.

Table 1: Basic characteristic of 240 LA NPC patients.

\begin{tabular}{|c|c|c|c|}
\hline Characteristic & Nimotuzumab & Non-nimotuzumab & $\mathrm{P}$ \\
\hline & No $(\%)$ & No $(\%)$ & \\
\hline Male & & & 0.073 \\
\hline Male & 88 (73.3) & 74 (61.7) & \\
\hline Female & $32(26.7)$ & $46(38.3)$ & \\
\hline Age (years) & & & 0.792 \\
\hline Range & $19-76$ & 18-77 & \\
\hline Median & 47 & 47 & \\
\hline$<50$ & $71(59.2)$ & $73((60.8)$ & \\
\hline$\geq 50$ & 49 (40.8) & $4739.2)$ & \\
\hline T stage * & & & 0.913 \\
\hline $\mathrm{T} 1$ & $4(3.3)$ & $4(3.3)$ & \\
\hline $\mathrm{T} 2$ & $16(13.3)$ & $20(16.7)$ & \\
\hline T3 & $54(45.0)$ & $52(43.3)$ & \\
\hline $\mathrm{T} 4$ & $46(38.4)$ & $34(28.3)$ & \\
\hline N stage * & & & 0.652 \\
\hline N0 & $11(9.2)$ & 15 (12.5) & \\
\hline N1 & $40(33.3)$ & $32(26.7)$ & \\
\hline N2 & $59(49.2)$ & $62(51.7)$ & \\
\hline N3 & $10(8.3)$ & $11(9.2)$ & \\
\hline Clinical stage * & & & 0.293 \\
\hline III & 67 (55.8) & $76(63.3)$ & \\
\hline IVA-b & $53(44.2)$ & $44(36.7)$ & \\
\hline IC regimen & & & 0.176 \\
\hline TPF & $42(35.0)$ & $27(22.5)$ & \\
\hline $\mathrm{TP}$ & $32(26.7)$ & $39(32.5)$ & \\
\hline GP & $4(3.3)$ & $7(5.8)$ & \\
\hline PF & $42(35.0)$ & $46(38.3)$ & \\
\hline AC & & & 0.420 \\
\hline No & $40(33.3)$ & 47 (39.2) & \\
\hline Yes & 80 (66.7) & $73(60.8)$ & \\
\hline
\end{tabular}

\section{Survival outcomes}

At the median follow-up duration of 56 months (range, 10-99 months), the estimated 5-year locoregional relapse-free survival (LR-RFS), distant metastasis-free survival (DMFS), progression-free survival (PFS), and overall survival (OS) rates were $92.1 \%, 90.1 \%, 84.4 \%$, and $90.2 \%$, respectively (Fig. 1 ).

The 5-year DMFS rate was significantly higher for patients treated with nimotuzumab than for those treated without nimotuzumab $(95.8 \%$ vs. $83.9 \%$, respectively; $\mathrm{p}=0.007 ;$ Figure $2 \mathrm{~B})$. There was a tendency to improve the 5 -year OS rate $(94.5 \%$ vs. $85.6 \%$, respectively) in the patients that received nimotuzumab, although the difference between the two groups didn't reach statistical significance $(p=0.0 .58 ;$ Figure 2D). Statistically significant differences in LR-RFS and PFS were not found between the two groups (5-year LR-RFS: 91.6\% vs. $91.1 \%$, respectively, $\mathrm{p}=0.957$, Figure 2A; 5-year PFS:
$87.4 \%$ vs. $81.3 \%$, respectively, $p=0.225$, Figure $2 C$ ).

\section{Failure mode.}

Treatment failure occurred in 37 patients (15.4\%) at last follow-up. In nimotuzumab group, 15 patients $(12.5 \%)$ experienced "any" failure (locoregional relapse occurred in 10 patients, distant metastases occurred in 5 patients), while in non-nimotuzumab group 22 patients experienced "any" failure ( locoregional relapse occurred in 5 patients, and locoregional relapse and distant failure occurred in 5 patient, distant failure alone was developed by12 patients). Patterns of treatment failure in NPC patients are listed in Table 2. Median time to failure for the nimotuzumab group versus non-nimotuzumab group was 18 months (range, 7 to 40 months) versus 27 months (range, 8 to 52 months), respectively.

Table 2 Treatment failure.

\begin{tabular}{llll}
\hline Failure mode & Nimotuzumab & Non-nimotuzumab & \multirow{2}{*}{0.020} \\
\cline { 2 - 3 } & $\mathrm{N}=120$ & $\mathrm{~N}=120$ \\
\hline Locoregional & 10 & 5 & \\
Locoregional and distant & 0 & 5 & \\
Distant & 5 & 12 & \\
Non-failure & 105 & 98 \\
\hline
\end{tabular}

\section{Identification of prognostic factors}

We performed multivariate analysis to evaluated the following potential prognostic factors: patient age, patient gender, clinical stage, adjusted tumor $(\mathrm{T})$ and lymph node $(\mathrm{N})$ stage, IC regimen, $\mathrm{AC}$, comorbidities, and nimotuzumab. Consistent with the results of univariate analysis, it showed that nimotuzumab could improve the survival outcomes of OS (HR, 2.650; 95\% CI, 1.075-6.530; $\mathrm{p}=0,034)$ and DMFS (HR, 4.032; 95\% CI, 1.482-10.968; $\mathrm{p}=0,006$ ) (Table 3).

Table 3: Multivariate analysis of prognostic factors in LA NPC patients.

\begin{tabular}{lllll}
\hline Endpoint & Characteristic & HR & $95 \%$ CI & $P$-value \\
\hline OS & With vs. without & 2.650 & $1.076-6.530$ & 0.034 \\
& nimotuzumab & & & \\
\multirow{2}{*}{ PFS } & III vs. IV* & 0.287 & $0.116-0.706$ & 0.007 \\
& III vs. IV* & 0.401 & $0.206-0.781$ & 0.007 \\
& Male vs. female & 2.691 & $1.119-6.472$ & 0.027 \\
& With or without & 3.022 & $1.068-8.549$ & 0.037 \\
\multirow{2}{*}{ LRRFS } & comorbidity & & & \\
DMFS & With vs. without & 4.032 & $1.482-10.968$ & 0.006 \\
& nimotuzumab & & & \\
& III vs. IV & 0.330 & $0.138-0.788$ & 0.013 \\
\hline
\end{tabular}

OS: Overall Survival. PFS: Progression-Free Survival. LRRFS: Locoregional Recurrence-Free Survival. DMFS: Distant Metastasis-Free Survival.

*The 7th AJCC/UICC staging system. 
$1 \mathrm{~A}$

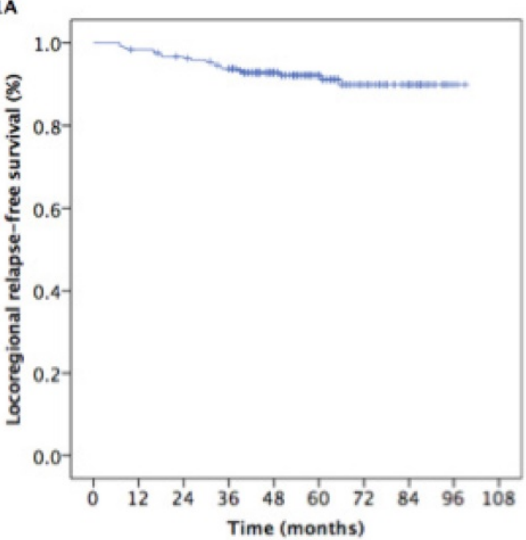

1C

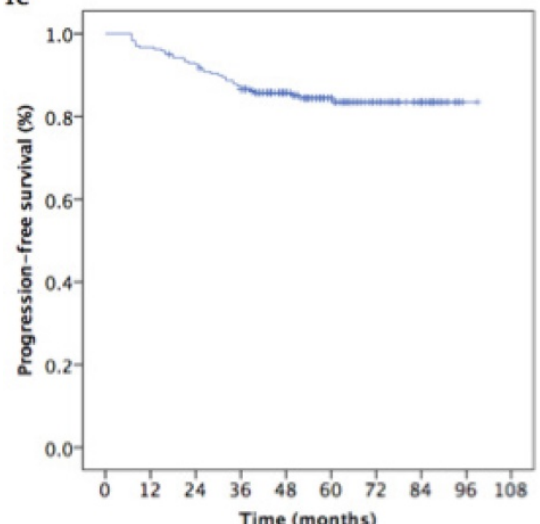

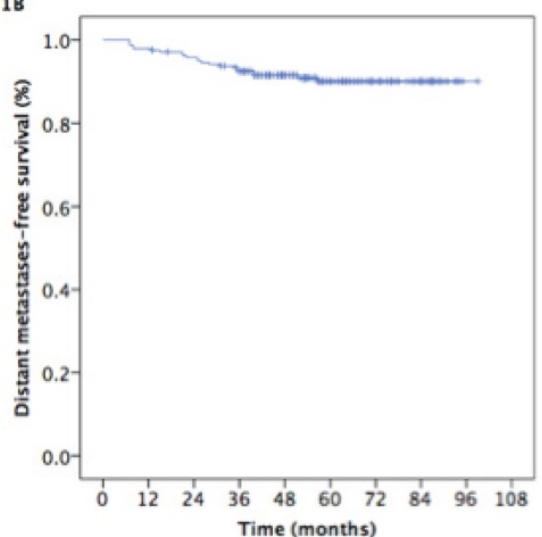

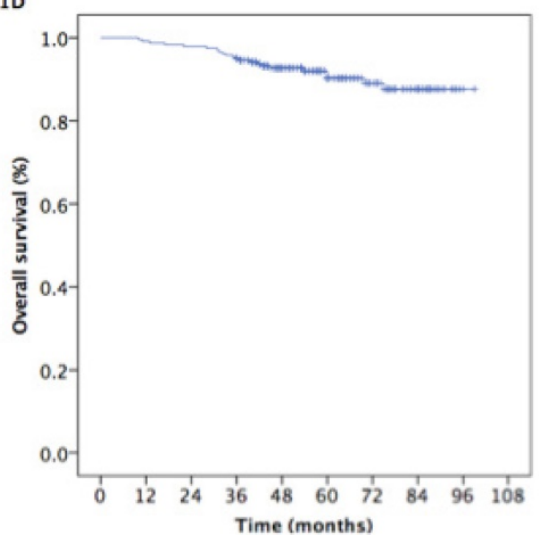

Figure 1: Kaplan-Meier estimates of the survival in 240 patients with nasopharyngeal carcinoma.
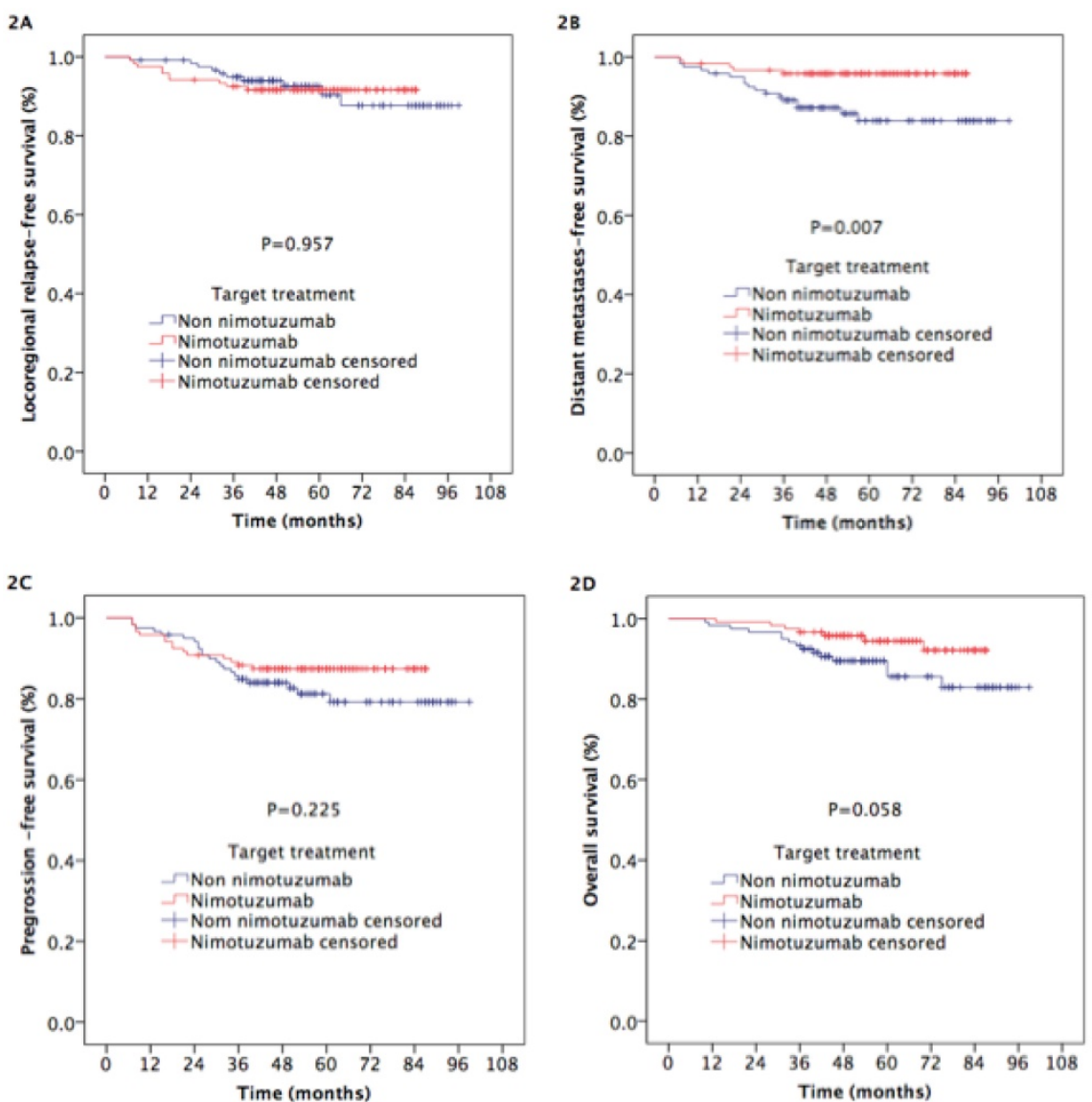

Figure 2: Kaplan-Meier estimates of the survival outcomes in nasopharyngeal carcinoma patients treated with nimotuzumab and without nimotuzumab. 

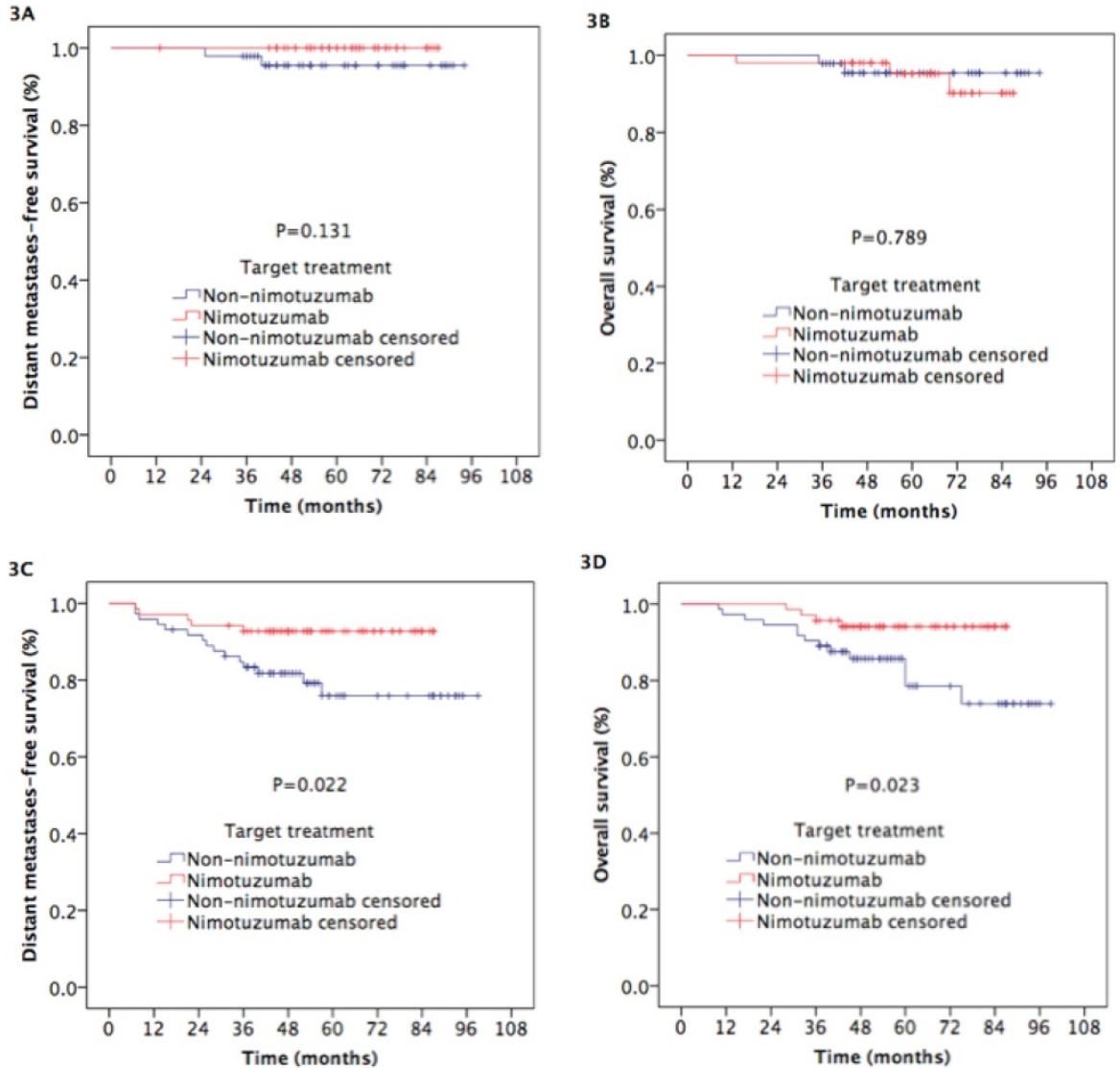

3D

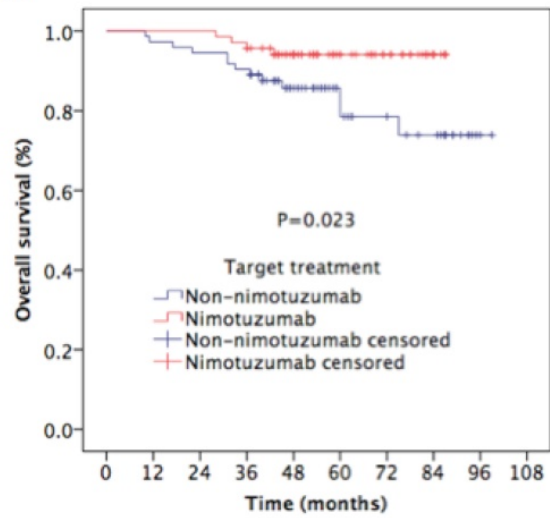

Figure 3: Kaplan-Meier estimates of the distant metastases-free survival and overall survival in nasopharyngeal carcinoma patients with N0-1 and N2-3 treated with nimotuzumab and without nimotuzumab.

\section{Subgroup analysis}

We performed the subgroup analysis to assess the prognostic value of nimotuzumab in the combination of IC plus CCRT according to $\mathrm{N}$ category and clinical stage. In patients with N0-1 category, the 5 -year LRRFS ( $94 \%$ vs. $90.3 \%$, respectively; $\mathrm{p}=0.623$ ), DMFS (100\% vs. $95.5 \%$, respectively; $\mathrm{p}=0.131$; Figure 3A), PFS (94\% vs. $88.3 \%$, respectively; $\mathrm{p}=0.398$ ) and OS (95.2\% vs. $95.4 \%$, respectively; $p=0.789$; Figure 3B) were comparable between the two groups. For patients with N2-3 category, the 5-year DMFS (92.7\% vs. $75.9 \%$, respectively; $\mathrm{p}=0.022$; Figure $3 \mathrm{C}$ ) and OS $(94.1 \%$ vs. $78.5 \%$, respectively; $p=0.023$; Figure 3D) rates in patients treat with nimotuzumab were higher than those in patients treated without nimotuzumab, while the differences of 5-year LRRFS $(89.8 \%$ vs. $94.3 \%$, respectively; $\mathrm{p}=0.747)$ and PFS $(82.5 \%$ vs. $76.7 \%$, respectively; $p=0.409$ ) between the two groups did reach statistical significance.

Likewise, for the patients with stage III NPC, the 5 -year LRRFS (93.9\% vs. 95.1\%, respectively; $\mathrm{p}=0.879$ ), DMFS (98.5\% vs. $87.9 \%$, respectively; $\mathrm{p}=0.040$; Figure 4A), PFS (92.4\% vs. $87.4 \%$, respectively; $\mathrm{p}=0.376)$ and OS (95.9\% vs. $89.3 \%$, respectively; $p=0.264$; Figure $4 \mathrm{~B})$ were comparable between the two groups. For patients with stage IVA-B, adding to IC plus CCRT improved 5-year DMFS (92.5\% vs. $75.8 \%$, respectively; $\mathrm{p}=0.030$; Figure $4 \mathrm{C})$ and OS $(92.6 \%$ vs. $78.6 \%$, respectively; $\mathrm{p}=0.054$; Figure $4 \mathrm{D})$, although the difference of OS between the two groups nearly reached statistical significance. But it did not significantly increase 5 -year LRRFS $(88.7 \%$ vs. $87.2 \%$, respectively; $\mathrm{p}=0.608)$ and PFS $(81.3 \%$ vs. $69.6 \%$, respectively; $\mathrm{p}=0.204$ ).

\section{Safety and toxicity.}

The safety was summarized by the number of patients experiencing any adverse event(s), which were collected by systematic assessment using terms from the CTCAE 3.0. The most commonly observed complications included hematologic and non-hematologic side effects. During the period of IC (Table 4), leukopenia and neutropenia in patients treated with nimotuzumab were reported as grade 3 and worse in severity in $34(28.3 \%)$ and 46 (38.3\%) patients respectively, while those adverse events in patients treated without nimotuzumab were reported in $35(29.2 \%)$ and $44(36.7 \%)$ patients, there were no significantly statistical differences between two arms $(p>0.05)$. The differences of other toxicities between two arms were not statistically significant. 
Table 4 Toxicity from IC between the two arms.

\begin{tabular}{|c|c|c|c|c|c|c|c|c|c|c|c|c|}
\hline \multirow[t]{2}{*}{ Adverse events } & \multicolumn{2}{|c|}{ Nimotuzumab } & \multicolumn{8}{|c|}{ Non-nimotuzumab } & \multirow[t]{2}{*}{ Z } & \multirow[t]{2}{*}{$\mathrm{P}$} \\
\hline & 0 & 1 & 2 & 3 & 4 & 0 & 1 & 2 & 3 & 4 & & \\
\hline \multicolumn{13}{|l|}{ Hematologic } \\
\hline Leukopenia & 10 & 31 & 45 & 23 & 11 & 8 & 39 & 38 & 22 & 13 & -0.862 & 0.389 \\
\hline Neutropenia & 12 & 24 & 38 & 20 & 26 & 14 & 26 & 36 & 16 & 28 & -0.329 & 0.742 \\
\hline Anemia & 83 & 26 & 8 & 3 & 0 & 86 & 22 & 10 & 2 & 0 & -0.353 & 0.724 \\
\hline Thrombocytopenia & 111 & 3 & 4 & 2 & 0 & 108 & 4 & 5 & 3 & 0 & -0.687 & 0.492 \\
\hline Liver function & 81 & 24 & 15 & 0 & 0 & 79 & 27 & 12 & 2 & 0 & -0.225 & 0.822 \\
\hline Renal function & 112 & 6 & 2 & 0 & 0 & 115 & 5 & 0 & 0 & 0 & $-0,877$ & 0.380 \\
\hline \multicolumn{13}{|l|}{ Non-hematologic } \\
\hline Mucositis & 105 & 10 & 5 & 0 & 0 & 107 & 11 & 2 & 0 & 0 & -0.459 & 0.646 \\
\hline Dermatitis & 114 & 6 & 0 & 0 & 0 & 117 & 3 & 0 & 0 & 0 & -1.017 & 0.309 \\
\hline Diarrhea & 106 & 10 & 3 & 1 & 0 & 112 & 6 & 2 & 0 & 0 & -1.349 & 0.177 \\
\hline Nausea/vomiting & 87 & 22 & 10 & 1 & 0 & 88 & 18 & 12 & 2 & 0 & -0.002 & 0.998 \\
\hline
\end{tabular}

Abbreviations: IC induction chemotherapy.

Table 5 Toxicity from CCRT between the two arms. Table 5 showed the incidence of acute adverse events by type and grade from CCRT. There were no significantly statistical differences in the hematologic, RT-related mucositis and dermatitis between two arms.

\begin{tabular}{|c|c|c|c|c|c|c|c|c|c|c|c|c|}
\hline \multirow[t]{2}{*}{ Adverse events } & \multicolumn{5}{|c|}{ Nimotuzumab } & \multicolumn{5}{|c|}{ Non-nimotuzumab } & \multirow[t]{2}{*}{ Z } & \multirow[t]{2}{*}{$\mathrm{P}$} \\
\hline & 0 & 1 & 2 & 3 & 4 & 0 & 1 & 2 & 3 & 4 & & \\
\hline \multicolumn{13}{|l|}{ Hematologic } \\
\hline Leukopenia & 43 & 46 & 23 & 8 & 0 & 44 & 40 & 24 & 12 & 0 & -0.373 & 0.709 \\
\hline Neutropenia & 37 & 42 & 26 & 13 & 2 & 39 & 41 & 27 & 10 & 3 & -0.281 & 0.779 \\
\hline Anemia & 100 & 14 & 6 & 0 & 0 & 96 & 19 & 5 & 0 & 0 & -0.603 & 0.547 \\
\hline Thrombocytopenia & 92 & 13 & 8 & 7 & 0 & 96 & 15 & 6 & 3 & 0 & -0.783 & 0.433 \\
\hline Liver function & 108 & 10 & 2 & 0 & 0 & 112 & 8 & 0 & 0 & 0 & -0.963 & 0.336 \\
\hline Renal function & 104 & 6 & 0 & 0 & 0 & 106 & 4 & 0 & 0 & 0 & -0.646 & 0.518 \\
\hline \multicolumn{13}{|l|}{ Non-hematologic } \\
\hline Mucositis & 0 & 43 & 63 & 12 & 2 & 0 & 47 & 58 & 14 & 1 & -0.373 & 0.709 \\
\hline Dermatitis & 0 & 103 & 12 & 5 & 0 & 0 & 99 & 18 & 3 & 0 & -0.625 & 0.532 \\
\hline Diarrhea & 108 & 7 & 4 & 1 & 0 & 109 & 8 & 3 & 0 & 0 & -0.259 & 0.796 \\
\hline Nausea/vomiting & 102 & 16 & 2 & 0 & 0 & 103 & 12 & 4 & 1 & 0 & -0.094 & 0.925 \\
\hline
\end{tabular}

Abbreviations: CCRT concurrent chemoradiotherapy.

\section{Discussion.}

With further research on the molecular mechanism of tumorigenesis and tumor development, targeted molecular therapy in patients with NPC will become the research focus. Overexpression of EGFR has been detected in $94 \%$ of patients with NPC [18]. Cetuximab is a common anti-EGFR monoclonal antibody drug. It has a good curative effect in the treatment of NPC, with a 2-year PFS of $86.5 \%$ to $89.3 \%$ and a 3-year OS of $90.9 \%$ [22], but severe oral mucositis and itchy acneiform rash limit its application in NPC. To minimize cetuximab-related toxicities, a novel EGFR-targeted agent without these toxicities is developed.

Nimotuzumab, a humanized immunoglobulin G1 (IgG1) isotype monoclonal antibody, with a unique safety profile and low skin toxicity, has been approved for the treatment of non-NPC HNSCC [16, 32]. The advantage of the drug is that the affinity constant is lower than that of cetuximab, allowing for high tumor uptake and low normal-tissue uptake [33]. Nimotuzumab requires bivalent binding for stable attachment, which makes the agent selectively bind to tumors with moderate-to-high EGFR levels. When EGFR expression is low, as in normal tissue, cetuximab still has high binding ability because of its higher affinity constant [33]. Our previous experiment confirmed that nimotuzumab sensitizes nasopharyngeal carcinoma cell line CNE-2 in vitro to RT and can reduce cancer cell proliferation, induce cell apoptosis, and change cell cycle distribution [34]. All these effects indicate that nimotuzumab plus RT can be utilized in the design of the clinical trial of NPC.

The extensive practical experience of the use of nimotuzumab in combination with radiotherapy in advanced NPC had started after the report of the phase II study of nimotuzumab plus RT for stage III-IVb NPC [35]. In this study nimotuzumab add-on group was superior to the placebo add-on group, resulting in a significantly higher complete remission rate $(90.63 \%$ vs $51.52 \%$, respectively, $\mathrm{p}=0.02)$ and higher 3 -year overall survival rate $(84.29 \%$ vs $77.61 \%$, respectively, $\quad \mathrm{p}<0.05) \quad$ without increasing radiation-related adverse events. 
$4 \mathrm{~A}$

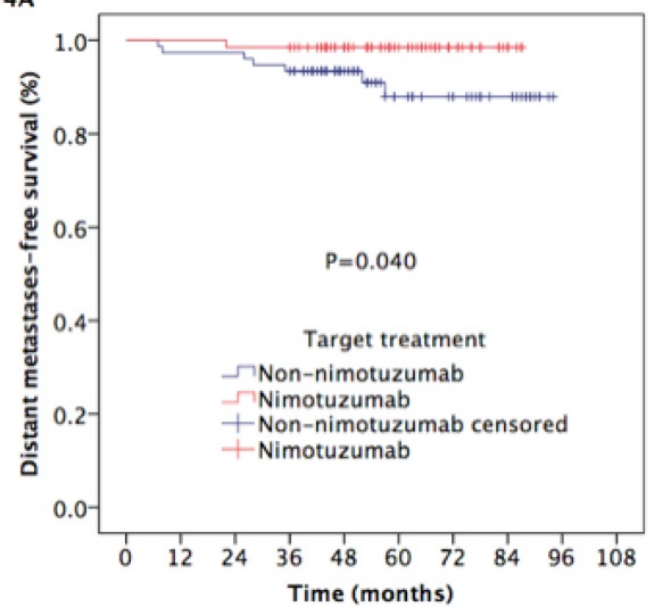

$4 \mathrm{C}$

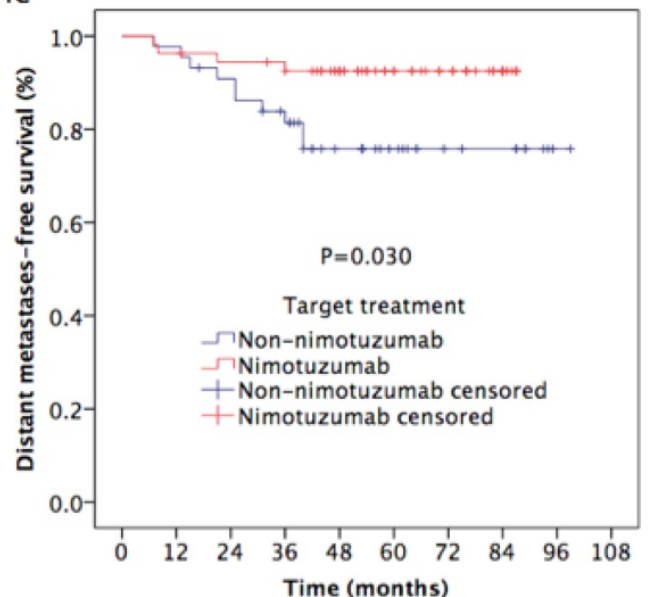

4B

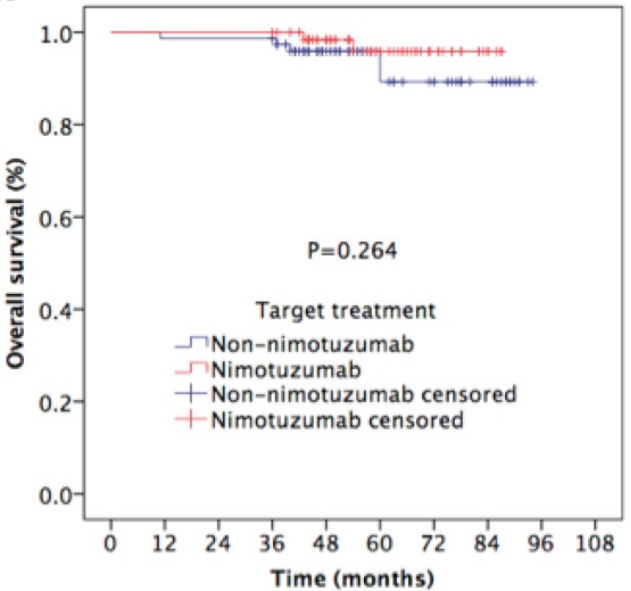

4D

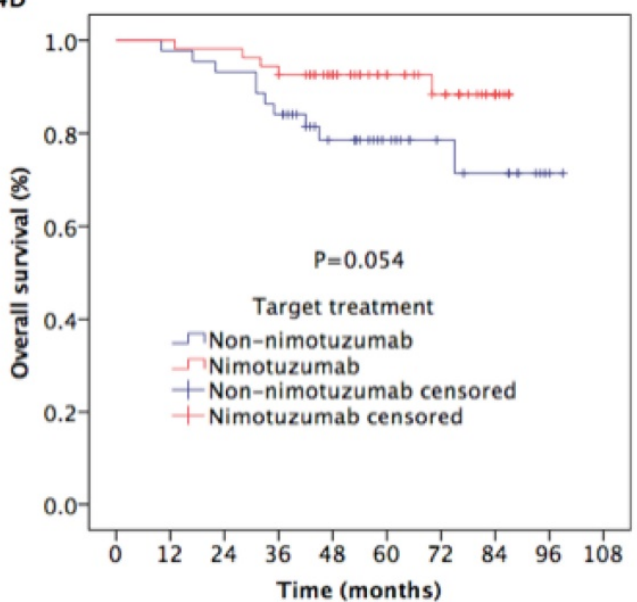

Figure 4: Kaplan-Meier estimates of the distant metastases-free survival and overall survival for stage III-IVB nasopharyngeal carcinoma patients treated with nimotuzumab and without nimotuzumab.

Table 6 Studies (Original research studies) on nimotuzumab in nasopharyngeal carcinoma advanced patients.

\begin{tabular}{|c|c|c|c|c|c|}
\hline Reference & Study Type & $\mathrm{n}$ & Treatment schedule & Survival benefits & Toxicity \\
\hline $\begin{array}{l}\text { Huang XD, et al. } \\
2007 \\
{[35]}\end{array}$ & Phase II & $\mathrm{N}=137$ & $\begin{array}{l}\text { Nimotuzumab in } \\
\text { combination with RT or } \\
\text { RT alone. }\end{array}$ & $\begin{array}{l}\text { 3-year OS rate }(84.29 \% \text { nimotuzumab group vs } \\
77.61 \% \text { non nimotuzumab group, } \mathrm{p}<0.05)\end{array}$ & $\begin{array}{l}\text { Most common nimo-related AEs were fever } \\
(4.28 \%) \text {, hypotension }(2.86 \%) \text {, nausea }(1.43 \%) \text {, } \\
\text { dizziness }(2.86 \%) \text {, rash }(1.43 \%) \text {. No worse } \\
\text { radiation-related AEs. }\end{array}$ \\
\hline $\begin{array}{l}\text { Rui-ping Zhai et } \\
\text { al. 2015. } \\
\text { [29] }\end{array}$ & Prospective open & $\mathrm{N}=38$ & $\begin{array}{l}\text { Nimotuzumab in } \\
\text { combination with IMRT. }\end{array}$ & $\begin{array}{l}\text { 3-year LRFS } 92.8 \% \text {, DMFS } 89.5 \% \text {, PFFS } 78.7 \% \text {, and } \\
\text { OS } 87.5 \% \text {. }\end{array}$ & $\begin{array}{l}\text { Grade } 3 \text { radiation-induced mucositis }(36.8 \%) \text {. } \\
\text { No skin rash and infusion reaction. }\end{array}$ \\
\hline $\begin{array}{l}\text { Li et al. } 2016 . \\
\text { [36], }\end{array}$ & $\begin{array}{l}\text { Matched } \\
\text { pair } \\
\text { retrospective }\end{array}$ & $\mathrm{N}=104$ & $\begin{array}{l}\text { TPF neoadjuvant } \\
\text { chemotherapy, followed } \\
\text { by IMRT plus concurrent } \\
\text { nimotuzumab or } \\
\text { cisplatin. }\end{array}$ & $\begin{array}{l}5 \text {-year OS and PFS for the nimo/RT vs. CDDP/RT } \\
\text { groups were } 63.9 \% \text { vs. } 81.4 \%(p=0.024) \text { and } 58.0 \% \\
\text { vs. } 80.6 \%(p=0.028) \text {. No significant difference for } \\
\text { OS in patients with stage II AJCC }(p=0.571) \text { or for } \\
\text { patients aged } 60 \text { years or older }(p=0.236) \text {. }\end{array}$ & $\begin{array}{l}\text { Nimotuzumab/RT } \\
\text { patients experienced less leukopenia and milder } \\
\text { nausea and vomiting with p values of } 0.048 \text { and } \\
0.000 \text {, respectively. }\end{array}$ \\
\hline $\begin{array}{l}\text { Jianfeng Huang, } \\
\text { et al. } 2017 \\
{[37]}\end{array}$ & Phase II & $\mathrm{N}=23$ & $\begin{array}{l}\text { Induction } \\
\text { chemotherapy followed } \\
\text { by IMRT plus, weekly } \\
\text { nimotuzumab and CCT. }\end{array}$ & 2-year PFSand OS were $83.5 \%$ and $95.0 \%$. & $\begin{array}{l}\text { Grade } 3-4 \text { oral mucositis dermatitis and } \\
\text { neutropenia occurred in } 7(36.8 \%), 1(5.3 \%) \text { and } \\
5(26.3 \%) \text { patients, respectively. } \\
1 \text { patient suffered } \\
\text { anaphylaxis, no acne-like rash was reported. }\end{array}$ \\
\hline $\begin{array}{l}\text { Zhi-gang Liu. } \\
2017 . \\
{[28]}\end{array}$ & Retrospective & $\mathrm{N}=42$ & $\begin{array}{l}\text { Nimotuzumab combined } \\
\text { with CCRT. }\end{array}$ & 2-year LRFS: $96.4 \%$, DMFS: $93.1 \%$ and OS: $96.6 \%$ & $\begin{array}{l}\text { Most common AEs were mucositis ( } 19 \text { patients), } \\
\text { hematology toxicity ( } 14 \text { patients) with } 6 \text { and } 3 \\
\text { cases of grade } 3 / 4 \text { toxicity respectively. Non } \\
\text { skin rash. }\end{array}$ \\
\hline $\begin{array}{l}\text { Fangzheng } \\
\text { Wang, et al. } \\
2017 \text {. } \\
{[38]}\end{array}$ & Retrospective & $\mathrm{N}=210$ & $\begin{array}{l}\text { Nimotuzumab plus } \\
\text { neoadjuvant } \\
\text { chemotherapy followed } \\
\text { by CCRT. }\end{array}$ & $\begin{array}{l}\text { 5-year LRFS: } 95.6 \% \text {, DMFS: } 91.7 \% \text {, PFS: } 84.0 \% \text {, and } \\
\text { OS: } 88.7 \% \text {, respectively. }\end{array}$ & $\begin{array}{l}\text { Grade } 3 / 4 \text { leukocytopenia } 24 \text { patients }(11.4 \%) \text {, } \\
\text { and } 6 \text { patients }(2.9 \%) \text { with mild liver damage } \\
\text { during neoadjuvant chemotherapy. Grade } 3 / 4 \\
\text { acute mucositis } 13 \text { patients }(6.2 \%) \text {, and } 12 \\
\text { patients }(5.7 \%) \text { with grade } 3 / 4 \text { leukocytopenia } \\
\text { during the CCRT. }\end{array}$ \\
\hline
\end{tabular}

OS: Overall Survival; LRFS: Local Recurrence-Free Survival; DMFS: Distant Metastasis-Free Survival; PFFS: Progression Failure-Free Survival; PFS: Progression-Free Survival; TPF: docetaxel + DDP $25 \mathrm{mg} / \mathrm{m} 2+5-\mathrm{fu}$. 
According to our knowledge, this is the first study to compare the survival benefits of the addition of IC before the combination of nimotuzumab plus CCT and intensity-modulated radiotherapy in patients with locoregionally advanced nasopharyngeal carcinoma. To date, only small-scale studies on adding nimotuzumab to RT or CCRT, for NPC patients have been conducted. Table 6 summarized the main results obtained from these original research studies.

In the retrospective paired study by Li et al [36], the OS and PFS rates for the nimotuzumab/RT treatment group were lower than those for cisplatin/RT treatment group, but in the stage II or the older than 60 years subgroups, no significant differences were seen for OS and PFS. Zhai et al [29] reported that the addition of nimotuzumab to IMRT showed promising locoregional control and survival outcomes for LA NPC patients. The estimated 3-year local recurrence-free survival, regional recurrence-free survival, distant metastasis-free survival, progression failure-free survival, and overall survival rates were $92.8 \%, 92.9 \%, 89.5 \%, 78.7 \%$, and $87.5 \%$, respectively.. Huang et al [37] and Liu et al [28] found that concurrent administration of nimotuzumab and CCRT yielded encouraging survival outcomes in LA NPC patients, with tolerable treatment-related toxicity. For the first two studies, because of the severe acute sequela of CCRT, nimotuzumab, as a preferred substrate for cisplatin, increased the quality of life in selected NPC patients, with similar treatment outcomes. However, in the last two studies, nimotuzumab added into the intensive modality of IC followed by CCRT improved the survival of LA NPC patients but with normal-tissue damage. The efficacy of nimotuzumab plus neoadjuvant chemotherapy followed by concurrent chemotherapy in locoregionally advanced nasopharyngeal carcinoma patients was found encouraging with a tolerable the toxicity profile, in a retrospective study conducted in our hospital between May 2008 to April 2014 [38]. Those outcomes will be the direction of further research.

This study combined the previous knowledge of the benefits of the combination of nimotuzumab with RT and CCRT but is the first to assess the prognostic value of this combination with the addition of IC in a large sample size and long follow up time, and the results of this research displayed that the addition of IC before nimotuzumab plus CCRT increased 5-year OS and DMFS for patients with locoregionally advanced NPC. However, nimotuzumab was not associated with better 5-year PFS and LRRFS.

In our study, we used PSM and multivariate analysis to assess the prognostic value of nimotuzumab for locoregionally advanced NPC. While the method of propensity score matching reduced the potential bias caused by the retrospective nature of the study, there are still some limitations of our research as the single center analysis and the heterogenicity of the chemotherapy regimens.

We found that nimotuzumab plus CCRT preceded by IC, in the treatment of locoregionally advanced NPC patients is effective. However, because of the retrospective nature of the study, our results should be regarded as preliminary and further prospective trials and multicentric studies are needed in the future to fully evaluate and confirm our results.

\section{Conclusion}

We observed that the administration of IC followed by nimotuzumab with CCRT in locoregionally advanced NPC patients showed promising clinic outcomes. Further randomized, controlled, multicenter phase III clinical trials are needed to confirm the therapeutic gain.

\section{Acknowledgements and funding}

This study was supported by grants from the Medical Science Foundation of Zhejiang Health Bureau (No. 2013KYB033, No. 2009B026, No. 2006A016, No. 2005B012, No. 2004B014), National Natural Science Foundation of China (No. 81502646, No. 81502647).

\section{Competing Interests}

The authors have declared that no competing interest exists.

\section{References}

1. LL Tang, WQ Chen, WQ Xue, et al. Global trends in incidence and mortality of nasopharyngeal carcinoma. Cancer Letters. 2016; 374: 22-30.

2. Chen L, Mao YP, Xie FY, et al. The seventh edition of UICC/AJCC staging system for nasopharyngeal carcinoma is prognostically useful for patients treated with intensity-modulated radiotherapy from an endemic area in China. Radiother Oncol. 2012; 104: 331-337.

3. Wee J. Nasopharyngeal carcinoma: a promising future. Lancet Oncol. 2012; 13: 116-118.

4. Lai SZ, Li WF, Chen L, et al. How does intensity modulated radiotherapy versus conventional two-dimensional radiotherapy influence the treatment results in nasopharyngeal carcinoma patients? Int J Radiat Oncol Biol Phys. 2011; 80: 661-668.

5. Al-Sarraf M and Reddy MS. Nasopharyngeal carcinoma. Curr Treat Options Oncol. 2002; 3: 21-32

6. Al-Sarraf M, LeBlanc M, Giri PG, et al. Chemoradiotherapy versus radiotherapy in patients with advanced nasopharyngeal cancer: phase III randomized Intergroup study 0099. J Clin Oncol. 1998; 16: 1310-1317.

7. Lee AW, Tung SY, Chua DT, et al. Randomized trial of radiotherapy plus concurrent-adjuvant chemotherapy vs radiotherapy alone for regionally advanced nasopharyngeal carcinoma. J Natl Cancer Inst. 2010; 102: 1188-1198.

8. Baujat B, Audry H, Bourhis J, et al. Chemotherapy in locally advanced nasopharyngeal carcinoma: an individual patient data meta-analysis of eight randomized trials and 1753 patients. Int J Radiat Oncol Biol Phys. 2006; 64: 47-56

9. OuYang PY, Xie C, Mao YP, et al. Significant efficacies of neoadjuvant chemotherapy and adjuvant chemotherapy for nasopharyngeal carcinoma by meta-analysis of published literature-based randomized, control trials. Ann Oncol. 2013, 24:2136-2146.

10. Chen YP, Guo R, Liu N, et al. Efficacy of the additional neoadjuvant chemotherapy to concurrent chemoradiotherapy for patients with 
locoregionally advanced nasopharyngeal carcinoma: a Bayesian network meta-analysis of randomized controlled trials. J Cancer. 2015; 6: 883-892.

11. Wang MM, Tian HM, Li G, et al. Significant benefits of adding neoadjuvant chemotherapy before chemoradiotherapy for locoregionally advanced nasopharyngeal carcinoma: a meta-analysis of randomized controlled trials. Oncotarget. 2016; 7: 48375-390.

12. Hui EP, Ma BB, Leung SF, et al. Randomized phase II trial of concurrent cisplatin-radiotherapy with or without neoadjuvant docetaxel and cisplatin in advanced nasopharyngeal carcinoma. J Clin Oncol. 2009; 27: 242-249.

13. Fountzilas $G$, Cluleanun $E$, Bobos $M$, et al. Induction chemotherapy followed by concomitant radiotherapy and weekly cisplatin versus the same concomitant chemoradiotherapy in patents with nasopharyngeal carcinoma: a randomized phase II study conducted by the Hellenic Cooperative Oncology Group (HeCOG) with biomarker evaluation. Ann Oncol. 2012; 23: 427-435.

14. Huang PY, Cao X, Mo HY, et al. A randomized trial of induction chemotherapy plus concurrent chemotherapy versus induction chemotherapy plus radiotherapy for locoregionally advanced nasopharyngeal carcinoma. Oral Oncol. 2012; 48: 1034-1044.

15. Wu F, Wang $\mathrm{R}, \mathrm{Lu} \mathrm{H}$, et al. Concurrent chemotherapy in locoregionally advanced nasopharyngeal carcinoma: treatment outcomes of a prospective, multicentric clinical study. Radiother Oncol. 2014; 112: 106-111.

16. Reddy BK, Lokesh V, Vidyasagar MS, et al. Nimotuzumab provides survival benefit to patients with inoperable advanced squamous cell carcinoma of the head and neck: a randomized, open-label, phase IIb, 5-year study in Indian patients. Oral oncology. 2014; 50: 498-505.

17. Herbst RS, Shin DM. Monoclonal antibodies to target epidermal growth factor receptor-positive tumors: a new paradigm for cancer therapy. Cancer. 2002; 94: 1593-1611.

18. Chua DT, Nicholls JM, Sham JS, et al. Prognostic value of epidermal growth factor receptor expression in patients with advanced stage nasopharyngeal carcinoma treated with induction chemotherapy and radiotherapy. Int J Radiat Oncol Biol Phys. 2004; 59: 11-20.

19. Kohler J, Schuler M. Afatinib, erlotinib and gefitinib in the first-line therapy of EGFR mutation-positive lung adenocarcinoma: a review. Onkologie. 2013; 36: 510-518.

20. Yewale C, Baradia D, Vhora I, et al. Epidermal growth factor receptor targeting in cancer: a review of trends and strategies. Biomaterials. 2013; 34: 8690-8707.

21. Bonner JA, Harari PM, Giralt J, et al. Radiotherapy plus cetuximab for locoregionally advanced head and neck cancer: 5-year survival data from a phase 3 randomised trial, and relation between cetuximab-induced rash and survival. Lancet Oncol. 2010;11:21-28.

22. Feng HX, Guo SP, Li GR, et al. Toxicity of concurrent chemoradiotherapy with cetuximab for locoregionally advanced nasopharyngeal carcinoma. Medical Oncology. 2014; 31: 170-8.

23. He X, Xu J, Guo W, Jiang $X$, et al. Cetuximab in combination with chemoradiation after induction chemotherapy of locoregionally advanced nasopharyngeal carcinoma: preliminary results. Future Oncol. 2013; 9: 1459-1467.

24. Talavera A, Friemann R, Gomez-Puerta S, et al. Nimotuzumab, an antitumor antibody that targets the epidermal growth factor receptor, blocks ligand binding while permitting the active receptor conformation. Cancer Research. 2009; 69: 5851-5859.

25. Crombet T, Osorio M, Cruz T, et al. Use of the humanized anti-epidermal growth factor receptor monoclonal antibody h-R3 in combination with radiotherapy in the treatment of locally advanced head and neck cancer patients. J Clin Oncol. 2004; 22:1646-1654.

26. Crombet $\mathrm{T}$, Torres $\mathrm{L}$, Neninger $\mathrm{E}$, et al. Pharmacological evaluation of humanized anti- epidermal growth factor receptor, monoclonal antibody h-R3, in patients with advanced epithelial-derived cancer. J immunother. 2003; 26: 139-148.

27. Du F, Zheng Z, Shi S, et al. S-1 and cisplatin with or without nimotuzumab for patients with untreated unresectable or metastatic gastric cancer: A randomized, open-label phase 2 trial. Medicine. 2015; 94: e958.

28. Liu ZG, Zhao Y, Tang J, et al. Nimotuzumab combined with concurrent chemoradiotherapy in locally advanced nasopharyngeal carcinoma: a retrospective analysis. Oncotarget. 2016; 7: 24429-24435.

29. Zhai RP, Ying HM, Kong FF, et al. Experience with combination of nimotuzumab and intensity-modulated radiotherapy in patients with locoregionally advanced nasopharyngeal carcinoma. OncoTargets and Therapy. 2015; 8: 3383-3390.

30. Austin PC. The relative ability of different propensity score methods to balance measured covariates between treated and untreated subjects in observational studies. Med Decis Making. 2009; 29:661-77.

31. Wang FZ, Jiang CE, Wang L, et al. Outcome and long-term efficacy of four facio-cervical fields conformal radiotherapy for nasopharyngeal carcinoma. Oncotarget. 2017; 8: 39756-39765.

32. Rodriguez MO, Rivero TC, Del CBR, et al. Nimotuzumab plus radiotherapy for unresectable squamous-cell carcinoma of the head and neck. Cancer Biol Ther. 2010; 9: 343-349.

33. Ramakrishnan MS, Eswaraiah A, Crombet T, et al. Nimotuzumab, a promising therapeutic monoclonal for treatment of tumors of epithelial origin. mAbs. 2009; 1: 41-48.
34. Hua $\mathrm{YH}, \mathrm{Ma} \mathrm{SL}, \mathrm{FU} \mathrm{ZF}$, et al. Effect of nimotuzumab on the radiation sensitivity of nasopharyngeal carcinoma cell line CNE-2. Chin J Zhejiang Med. 2011; 33: 836-839.

35. Huang XD, Xu GZ, Gao L, et al. A phase II study of (Clinical study of) anti-epidermal growth factor receptor monoclonal antibody h-R3 combined with radiotherapy in the treatment of advanced nasopharyngeal carcinoma[J]. Chin J Oncol, 2007, 29(1): 197-201.

36. Li HM, Li P, Qian YJ, et al. A retrospective paired study: efficacy and toxicity of nimotuzumab versus cisplatin concurrent with radiotherapy in nasopharyngeal carcinoma. BMC Cancer. 2016; 16: 946-55.

37. Huang JF, Zhang FZ, Zou QZ, et al. Induction chemotherapy followed by concurrent chemoradiation and nimotuzumab for locoregionally advanced nasopharyngeal carcinoma: preliminary results from a phase II clinical trial. Oncotarget. 2017; 8: 2457-2465.

38. Fangzheng W, Chuner J, Zhimin $Y$, et al. Efficacy and safety of nimotuzumab with neoadjuvant chemotherapy followed by concurrent chemoradiotherapy for locoregionally advanced nasopharyngeal carcinoma. Oncotarget. $2017 \mathrm{Apr}$ 21. 